\title{
The management of acute low back pain in adults: a guide for the primary care physician, Part II
}

\author{
Brighton SW, Former Head \\ Department of Rheumatology, Steve Biko Academic Hospital, University of Pretoria \\ Correspondence to: Stan Brighton, e-mail: brighton@telkomsa.net \\ Keywords: chronic back pain, acute low back pain (LBP), spinal stenosis, radiculopathy
}

\begin{abstract}
When a patient presents with acute low back pain (LBP), any red flag warnings of serious disease should first be excluded. Yellow and blue flag warnings of psychological factors should be noted. A psychological opinion of patients with substantial psychological distress could be sought. Advice may be offered on the benign nature of non-specific LBP. The person should be encouraged to be physically active and to continue with normal activities as far as possible. A structured exercise programme, that includes aerobic activity, movement instruction, muscle strengthening, postural control and stretching, should be devised. A combined exercise and psychological treatment programme that includes a cognitive behavioural approach can be considered in patients with significant disability or substantial psychological distress. A course of acupuncture may also be added. Manual therapy, including spinal manipulation, could be considered. Paracetamol should be the first medication option. If this is inadequate, a nonsteroidal anti-inflammatory drug or weak opioid, or both, can be added. Individual risks for side-effects and the patient's preference should be taken into account. Strong opioids should be considered in patients in severe pain, but for short-term use only. Antidepressants and gabapentine or pregabolin can be considered when there is a neurogenic component of the pain. Consider obtaining a surgical opinion on patients who have completed an optimal package of care and who still have persistent severe non-specific LBP. Progressive neurological fallout requires a surgical opinion.
\end{abstract}

(P) Peer reviewed. (Submitted: 2012-04-14. Accepted: 2012-02-03.)@ Medpharm

S Afr Fam Pract 2012;55(1):26-32

\section{Introduction}

Chronic low back pain (LBP) is a hetrogeneous condition with multiple aetiologies that can give rise to pain, by definition of a duration that is longer than three months. Chronic LBP is not a diagnosis or a clinical entity in its own as there are patients with different stages of disability, chronicity and impairment. Prognostic factors and an assessment of the degree of impairment during daily living are essential in the assessment of patients. A number of evidence-based therapies are usually sufficient in the case of little impairment or disability.

From an aetiological point of view, chronic back pain can be divided into two broad groups: nociceptive and neurogenic pain.

Nociceptive pain arises from direct tissue injury for whatever reason, for example trauma or infection. As a result of the tissue injury, proinflammatory cytokines and other neurotransmitters are released, which in turn stimulate the somatosensory system (pain pathways). Nociceptive pain diminishes with the natural healing process of the primary lesion.

On the other hand, neurogenic pain arises as a consequence of a lesion or disease that directly affects the somatosensory nervous system and continues long after the natural healing of the primary lesion has taken place. ${ }^{1}$ Neuropathic pain can arise from damage to nerve pathways at any point from the terminals of the peripheral nociceptives to the cortical neurons in the brain. In some cases, pain impulses can be generated in the nerve pathways themselves, long after the primary lesion has healed. This can make therapeutic decisions very difficult as procedures aimed at the original triggering lesion are inappropriate because of a miguided belief as to the cause of the pain.

The exact prevalence of neuropathic pain is not known, but a recent study reported that $35 \%$ of patients with prolonged LBP have neuropathic pain predominantly. ${ }^{2}$

To further complicate the diagnosis, both neuropathic and nociceptive pain can coexist, but an attempt should be made to identify the different main components and to treat each of them according to the best available evidence. An example would be neurogenic pain that results from chronic disc degeneration, but with ongoing nococeptive pain as a result of continuous leakage of nucleuc palposus material (leaky disc syndrome).

Neuropathic pain should be considered when the pain continues beyond the expected natural healing phase of the primary lesion. It can be spontaneous or provoked pain with pricking, tingling, pins and needles, electric shocks 
with shooting pain, a hot or burning sensation, numbness and pain evoked by light touch or a painful cold or freezing sensation. ${ }^{3}$

In many cases, there is complete recovery after surgical decompression of the nerve or nerve root. Unfortunately, a small group do not recover. Treatment options are complicated. Such patients should be referred for specialist opinion.

Disc herniation is implicated in $45 \%$ of patients, especially in younger patients. Facet joint pain, particularly osteoarthritis of the paravertebral joints, is implicated in $40 \%$, and becomes an increasingly important cause of pain with ageing. Dural nerve or root pain and the dorsal root ganglion play an important role in $12 \%$ of patients' chronic LBP. The sacroiliac joint is implicated in up to $18 \%$ of cases, particularly in failed back surgery after lumbar fusion and the stress that is imposed on this joint. ${ }^{4}$

Psychological and social factors may have a profound biasing influence on all aspects of LBP. These vary from a minor to major role in the patients' response to the pain impulse from the underlying disease process, and their response to treatment. ${ }^{5}$ Genetic factors have been found to play an important role in disc degeneration. ${ }^{6}$ No single intervention has been shown to be more effective in treating patients with a more substantial disability. This is because of pain's multidimensional nature. ${ }^{7}$

\section{History}

A detailed chronological history of the pain should be obtained. When did the first pain occur? Was there any initiating factor? A detailed description of the pain must then be recorded, including its nature, e.g. shooting, stabbing, pins and needles; its frequency and its intensity on the 1-10 visual analogue scale.

Particular inquiry must be made into the possibility of any serious underlying pathology, the so-called "red flag" warnings. ${ }^{8}$ These include a history of cancer, unexplained weight loss, immunosuppression and a history of intravenous drug use: either illicit or intravenous drips. Other red flag warnings are prolonged steroid usage (past or present), bladder infections, significant trauma, a fall or minor trauma in a potentially osteoporotic or elderly individual, walking limitations because of leg pain, persistent numbness or weakness in the legs, signs of possible chronic cauda equina syndrome (including bladder dysfunction), and any systemic disease. These indicate the possibility of a serious underlying pathology that could possibly be the cause of the back pain and require further investigation.

The history must include an inquiry into possible "yellow flag" or "blue flag" warnings. ${ }^{9}$

These flag warnings indicate possible psychological or social factors that may play a role in the patient's response to their pain. Psychosocial factors have a profound biasing influence on all aspects of LBP behaviour, including the reporting of it and response to treatment. They can contribute to delayed recovery from acute back pain or progression to a chronic pain pattern.

The most common psychosocial yellow flag factors are:

- Advanced age

- Lower income group

- Severe multifocal pain

- Depression

- Higher functional disability

- Domestic issues.

Blue flag warnings refer to the possibility of work and employment-related factors that can materially affect the reporting and experience of pain.

The most common work and employment-related blue flag factors are:

- Heavy, unpleasant or dangerous work

- Job dissatisfaction

- Adversarial job relations

- Possible disability or compensation issues

- Previous sick listing (time off work).

Empathy, patience and tact are often required to elicit many of these problems. Sensitive or embarrassing situations may be withheld from the examiner. Although the experienced clinician should be able to obtain useful information, at times, a professional psychologist may be required.

\section{Examination}

\section{Physical examination}

A detailed physical and particular neurological examination is required in a systematic search for possible lesions that would be amenable to correction by invasive procedures. Serious underlying conditions, the so-called red flag warnings, must be excluded.

In 1980, Waddell et a ${ }^{10}$ published a series of signs, called nonorganic signs to help identify patients who required a more detailed psychological evaluation because of yellow and blue flag warnings. These signs are still widely used and have been well validated. If any of these signs are positive, it is an indication that psychological factors may be present in the patient's pain picture.

A standardised group of five physical signs has been developed:

1. Tenderness: Usually, tenderness that relates to physical disease is localised to a particular structure or area. Nonorganic tenderness may be superficial. There could be tenderness in response to a light pinch over a wide area of the lumbar spine and not localised to any particular area or muscular group.

2. Simulation: Simulation tests give the patient the impression that a particular examination is being carried out, when in fact it is not. With the patient standing erect, press down on the patient's head. This should cause no increase in back pain. This does not apply to neck pain. If the patient claims that it increases the back pain, it is regarded as a positive test. Alternately, stand behind 
the patient gripping his or her hips and then rotate the patient to the left and to the right (pivoting the patient on the ankles) keeping the shoulders and pelvis in the same plane. If this causes pain, it is a positive sign.

3. Distraction: Distraction tests divert the patient's attention while a test is carried out on what the patient reported to be painful. Ask the patient to sit with his or her lower legs hanging down and then distract the patient's attention by pretending to examine the ankle reflexes, while at the same time slowly straightening the leg.

4. Regional disturbances: The essential feature of this test is divergence from accepted neuroanatomy, either weakness or sensory changes that do not follow normal anatomical patterns.

5. Overreaction: This is self-explanatory.

In terms of scoring, if three or more of the five tests receive a positive response, then it is considered to be significant and to possibly indicate that an underlying psychological factor is contributing to the pain. Isolated positive signs must be ignored.

\section{Examination of the spine}

Spinal range of motion is of limited diagnostic value and contributes little to therapeutic choices. Pain that radiates to the buttocks or leg with side-bending or a difference of more than 20 degrees in straight leg raising on either the left or right side indicates a longer recovery time. ${ }^{11}$

Referred pain from musculature trigger points may mimic sciatica and is called pseudoradiculopathy. Deep palpation of the lumbar musculature should be undertaken in a search for painful fibrocytic nodules. ${ }^{12}$

\section{Functional status}

Once serious spinal pathology and nerve root pain have been excluded, the patient's functional status must be considered. Those with minimal functional impairment can be presumed to be suffering from simple LBP. Simple LBP constitutes $90 \%$ of chronic back pain that is seen in general practice, serious spinal pathology (8\%) and nerve root compromise (2\%). ${ }^{11,13}$

\section{Special investigations}

It it well established that there is a poor correlation between back pain and X-ray findings. ${ }^{14}$ In the absence of red flag warnings and the normally benign course of non-specific LBP, the Royal College of Radiologists recommends that an X-ray should be reserved for: ${ }^{15}$

- Nonresolving symptoms (more than 4-6 weeks)

- Deteriorating symptoms

- Neurological signs

- History of trauma.

Advanced imaging [magnetic resonance imaging (MRI) or computed tomography (CT) scanning] should be reserved for patients when surgery is being considered, or for those in whom systemic disease is strongly suspected. MRI is the best imaging procedure for patients with radicular symptoms or for those with red flags or suspected serious disease. ${ }^{16}$

Frequently, further advanced imaging identifies many radiological abnormalities that poorly correlate with symptoms. ${ }^{17,18}$ Clinicians should be aware that findings on MRI or CT scans, such as bulging discs without nerve impingement, are often non-specific. Further decisions should be based on the clinical correlation between the symptoms and the radiographic findings, as well as the severity of symptoms. Studies on the natural history of MRI-diagnosed disc abnormalities have shown that after five years, psychological factors and physical work characteristics were more powerful that MRI in predicting future pain and work incapacity.

\section{Management}

LBP is a hetrogeneous condition with multiple aetiologies that can give rise to pain. Treatment goals include:

- Reducing pain intensity.

- Maintaining physical activity.

- Maximising functional abilities, despite persistent pain.

- Returning to work, or continuing to have a normal lifestyle.

- Avoiding inappropriate medications or treatments.

- Increasing the patient's ability to self-manage his or her pain.

A number of evidence-based therapies are usually sufficient for patients with little impairment or disability in their daily activities. Frequently a multidisciplinary management programme, together with the contribution of other health professionals, may be required.

\section{The biopsychosocial model}

The management of chronic LBP has moved away from the biomedical model of a specific pathology that is amenable to a specific "cure" to the comprehensive biopsychosocial model which places an emphasis on the restoration of function. ${ }^{19}$ It is now recognised that psychosocial factors, such as emotional stress, can impact on the reporting of symptoms, medical disorders and response to treatment. The biopsychosocial model focuses on both disease and illness. Disease is defined as an objective biological event. In contrast, illness refers to a subjective experience or selfattribution that a disease is present. Thus, illness refers to how the patient and members of his or her family live with and respond to the symptoms of the disability. When treating people with chronic pain, mood states must be taken into account, as well as the physical pathology and somatic factors. Pain cannot be treated successfully without attending to the patient's emotional state. The yellow and blue flag warnings of possible psychological factors, as explained above in the section on history taking, should be further explored.

Cognitive behavioural therapy is increasingly being used to address maladaptive behaviours, beliefs and negative thinking in patients. ${ }^{20,21}$ Anxiety, depression, anger, a 
negative mood and catastrophising and feared avoidance beliefs, might all contribute to the pain experience. The individual's interpretation, evaluation and beliefs about his or her health condition affect the degree of emotional and physical disability that is associated with the pain.

\section{Provision of patient information}

This is an important part of the treatment. Accurate information should be given to the patient, as many myths and prejudices exist. ${ }^{22}$

Once serious disease has been excluded, it should be explained to the patient that there is little likelihood of serious complications and that the term "simple back pain" is commonly used. Provide the patient with the most safe and effective methods of symptom control. Give guidance on activity modifications and on which activities are safe to perform. Detail methods to help to limit the recurrence of the pain. If the symptoms persist, then explain the effectiveness and risks of further diagnostic tests to the patient.

\section{Pharmacological treatment}

Medication for pain control must only be part of a comprehensive management plan. ${ }^{3}$ An attempt should be made to differentiate between nococeptive and neuropathic type pain, but in practice, there is often a considerable overlap between the two. As a general rule, flares of pain against a background of chronic pain in older patients with degenerative facet joints is more likely to be nociceptive pain and amenable to simple analgesics.

\section{Analgesics}

Paracetamol should be the preferred first-choice medication for LBP because of the evidence of its effectiveness and the low risk of side-effects. If adequate doses of paracetamol are inadequate, nonsteroidal anti-inflammatory drugs (NSAIDs) may be started. A combination of NSAIDs and mild opioids, e.g. codeine or tramadol, or a combination of NSAIDs and muscle relaxants, may be used.

The use of weak opioids, e.g. tramadol, can be recommended in patients with non-specific chronic LBP who do not respond to other modalities. Opioid analgesics are an option in patients with severe, disabling pain that is not controlled by paracetamol, NSAIDs or tramadol. The potential benefits and harm of taking opioid analgesics should be carefully weighed before starting therapy because of the substantial risks. Failure to respond to a time-limited course of the opioid should lead to full reassessment and consideration of alternative therapies. ${ }^{23}$

Pharmacological management remains the most important therapeutic option for chronic neuropathic pain. ${ }^{24}$

\section{Antidepressants}

The tricyclic antidepressants, e.g. amitriptyline, have been shown to give moderate relief to various neuropathic pain conditions. They are relatively well tolerated in low doses, but higher doses are often indicated. ${ }^{25}$
The serotonin noradrenergic reuptake inhibitors, e.g. duloxetine and venlafaxine, have been shown to be effective in relieving pain in patients with chronic neurogenic LBP. Frequently, they are considered as co-medication with other modalities for pain relief.

The selective serotonin reuptake inhibitors have a weak analgesic effect only, and the clinical relevance of these compounds is questionable.

\section{Anticonvulsants}

Both gabapentine and pregabolin have been shown to be effective across different peripheral and central neuropathic pain conditions. However, no overall evidence of superior efficacy has been attributed to either of these drugs. ${ }^{26}$ Other antiepileptic drugs may have a pain-relieving effect, but side-effects are a significant problem.

\section{Drug combinations}

Drug combinations are often used because of the limited efficacy of available treatments. Various commercial combinations of analgesics and NSAIDs are frequently used that provide relief in mild to moderate cases.

\section{Physical treatments}

\section{Bed rest}

Patients with a severe flare of pain can be treated with a short period of bed rest and mobilised as soon as possible thereafter. The shorter the period of bed rest and the sooner mobilisation begins, the better. Bed rest is not a therapy for chronic pain. ${ }^{27}$

\section{Activity and exercise}

Exercise forms a crucial part of the management of the patient with chronic LBP. ${ }^{28}$ Activities that are known to increase the mechanical stress on the spine should be avoided. In particular, lifting, prolonged sitting (particularly in an unsupported chair), as well as bending or twisting, should be avoided. Specific instructions in this regard can be given by other health workers, e.g. physiotherapists or biokineticists.

The patient who has an occupation that places considerable mechanical stress on the back should be carefully evaluated. This often necessitates a review of the working conditions. Many factories may employ an occupational therapist, who if available, should be consulted. Even temporarily placing the patient in a job that has less mechanical strain is better than booking him or her off work completely. The longer a person is booked off work, the greater the risk of him or her not returning to work.

There is no evidence that any particular type of exercise is more effective than another. Subgroups of patients may respond differently to various types of exercise. Strategies by the patient to improve adherence are important. Patient preferences and expectations should be considered when deciding which type of exercise to choose. 
Exercise programmes that minimally stress the back (walking; bicycle riding, with the handle bars positioned high enough so as to straighten the back, and swimming) can confidently be prescribed. Simple conditioning exercises for the trunk muscles, especially back extensors, if gradually increased, are helpful. Initially, these exercises may aggravate symptoms before relief is felt. No evidence exists for involved exercise programmes beyond these simple exercises. Physiotherapists and biokineticists can provide a valuable contribution to treatment. Regular supervised exercise programmes give superior results.

\section{Spinal manipulation}

Manipulation can be effective in hastening recovery. ${ }^{29}$ There is insufficient evidence for or against manipulation in patients with radiculopathy, but anecdotal evidence is in favour. Many dramatic recoveries have been described. Any exacerbation of symptoms is seen to be a contraindication to continuing.

\section{Other modalities}

A course of acupuncture has been shown to be of moderate benefit in selected patients. ${ }^{30}$ There is no objective evidence that spinal traction is of any benefit. There have been a number of reports of aggravation of sciatica following traction. This was attributed to traction of the nerve root over a bulging disc.

A review of the extensive literature on other physical agents and modalities, including ice, heat, diathermy, ultrasound, cutaneous laser, electrical stimulation and transcutaneous electrical nerve stimulation, has revealed inconsistent results. ${ }^{31,32}$ Frequently, patients with palpable trigger points and nodules in the lumbar and gluteal musculature respond to these treatments. Massage to these areas also gives relief, although of a more temporary nature. ${ }^{33}$ However, these are all passive treatments. The basic principle of back pain treatment is to encourage active patient participation.

\section{Lumbar corsets and back belts}

There is little objective evidence that lumbar corsets and back support belts are of use, but the placebo effect is strong in some patients. Many patients with severe degenerative changes of the lumbar spine feel more secure with a support, e.g. a corset. Anecdotal evidence is that male patients who carry out bending work feel more comfortable using simple supports such as a weightlifter's belt.

\section{Invasive procedures}

\section{Epidural steroids}

There is conflicting evidence on the effectiveness of epidural steroids in treating non-specific LBP. They provide short-term relief to a minority of patients with radicular symptoms. ${ }^{34}$

\section{Facet-joint injections}

There have been few controlled trials on the use of intraarticular facet joint steroids, but they may well be effective in the older patient with osteoarthritic degeneration of the joints.

\section{Surgery}

Fusion surgery cannot be recommended unless two years of all other recommended conservative treatments have failed in patients without radicular pain or nerve involvement. Considering the high complication rates of surgery, as well as the costs to society and suffering that is experienced by patients with failed back surgery, only carefully selected patients with severe pain should be considered for this procedure. $^{33,35}$

Surgery should be considered earlier in the case of:

- Disabling sciatica

- Further nerve fallout, with significant or progressive neurological deficit, and with motor deficits at more than one level.

A number of other invasive procedures can be considered in the patient with intractable pain and who has not responded to the abovementioned conservative approach.

Radiofrequency denervation of the nerves that supply the facet joints is superior to placebo procedures. These findings apply only to those studies in which patients were first selected by local anesthetic blocks. ${ }^{36}$ In properly selected patients, radiofrequency denervation is highly effective, but the main disadvantage is the limited duration of action of approximately six months and also the high costs of hospitalization, theatre, anaesthetic and radiologist fees.

Pulsed radiofrequency is less effective than radiofrequency denervation in treating pain that arises from the lumbar facet joints. Proper selection of patients and optimal technique are important to achieve optimum results.

\section{Case study}

A 56-year-old businessman presented with a history of chronic LBP that had become progressively worse over several months. The pain was minimal in the mornings, developed into an ache during the day, and increased in severity towards the evening. He said that his round of golf on Saturdays loosened his back, but that when he cooled down, the pain worsened. He had been seen by other healthcare professionals and was prescribed anti-inflammatories. He had also had three courses of physiotherapy. He maintained that he had experienced little relief. He then had an epidural infiltration, which again provided temporary relief only. Plain X-rays showed degenerative changes and disc-space narrowing at L2, L3 and L4, with scoliosis (Figure 1). An MRI was carried out which reported annular bulging at $L 2$ and $L 3, L 3$ and $L 4$ and $L 5 / S 1$ levels, and exit foramina impingement at L3 and L4 and L5 and S1 levels.

He was advised to consider a surgical option, but was very reluctant to do so because of work commitments. His family practitioner suggested a second opinion. 


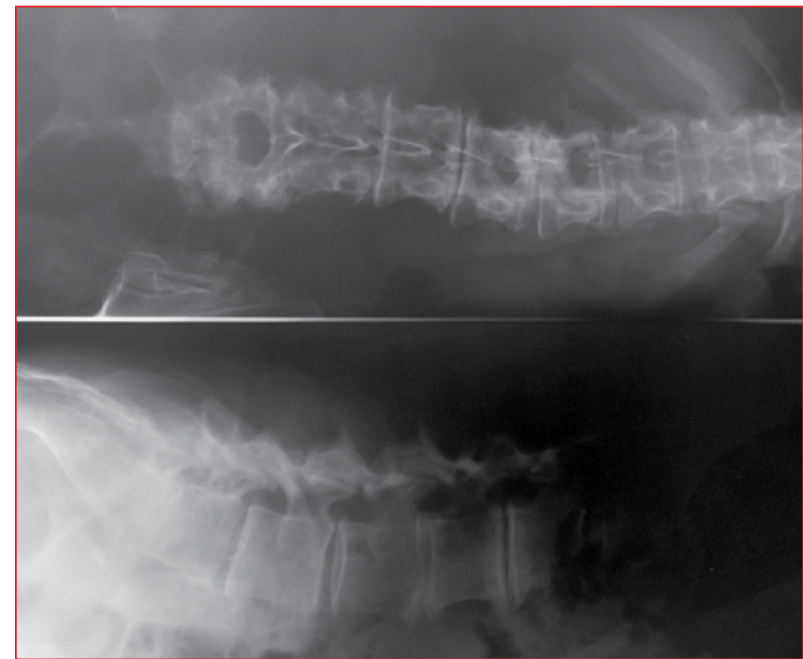

Figure 1: X-rays showed degenerative changes and disc-space narrowing at L2, L3 and L4, with scoliosis

On examination, he was found to be neurologically intact, with 70-degree bilateral straight-leg raising and slightly restricted lumbar extension and side-bending. Initially, there were no yellow flag warnings of possible psychological causes, but three of the Waddell nonorganic signs were positive. This indicated that there might be some psychological aspect to his pain. Initially, he was treated with nerve blocks to the lower lumbar region, but with limited relief. After further questioning, he admitted that there were domestic problems and tactful questioning gave a possible explanation. He had slowly developed erectile dysfunction that caused him great embarrassment. He was prescribed sildenafil. At a follow-up visit a week later, he expressed utter amazement that he had considered his back pain to be genuine, rather than a mask for something else. A structured programme of exercises under the guidance of a biokineticist, instructions on back care and a prescription for sidenafil for use "as needed" has reduced his pain to discomfort with which he is able to live.

This case illustrates the powerful role that psychological factors can play in all aspects of LBP and the difficulty in eliciting these underlying factors, and in his case, catastrophising what was otherwise fairly mild discomfort.

\section{References}

1. Treede RD, Jensen TS, Campbell JN, et al. Redefinition of neurogenic pain and a grading system for clinical use: consensus statement on clinical and research diagnostic criteria. Neurology. 2008;70(18):1630-1635.

2. Freyenhagen R, Baron R, Gockel U, Tolle TR. painDETECT: a new screening questionnaire to identify neuropathic components in patients with back pain. Curr Med Res Opin. 2006;22(10):1911-1920.

3. Chou R, Qaseem A, Snow V, et al. Diagnosis and treatment of low back pain: a joint clinical practice guideline from the American College of Physicians and the American Pain Society. Ann Intern Med. 2007;147(7):478-491.

4. Pang WW, Mok MS, Lin ML, et al. Application of spinal pain mapping in the diagnosis of lowbackpain. Acta Anaesthesiol Sin. 1998:36(2):71-74.

5. Jones EA, McBeth J, Nicholl B, et al. What characterises persons who do not report musculoskeletal pain ? Reults from a 4-year population based longitudinal study (the Epifund study). J Rheumatol. 2009;36(5):1071-1077.

6. Battie MC, Videman T et al. Heritability of low back pain and the role of disc degeneration. Pain 2007;131(3):272-280

7. Airaksinen O, Brox Jl, Cedraschi C, et al. European Guidelines for the management of chronic non-specific low back pain. Eur Spin J, 2006;Suppl 2:S192-S300.

8. Henschke N, Maker CG, Refshauge KM, et al. Prevalence and screening for serious spinal pathology in patients presenting to primary care settings with acute low back pain. Arthritis Rheum. 2009;60(10):3072-3080.

9. Pincus T, Burton AK, Vogel S, et al. A systematic review of psychological factors as prediction of chronicity/disability in cohorts of low back pain. Spine (Phila Pa 1976). 2002;27(5):E109-R120.

10. Waddell G, McCulloch JA, Kummel E, Venner RM. Nonorganic physical signs in low-back pain. Spine (Phila Pa 1976). 1980;5(2):117-125.

11. Last AR, Hulbert K. Chronic low back pain: evaluation and management. Am Fam Phys. 2009;79(12):1067-1074.

12. Borg-Stein J, Wilkins AN. Soft tissue determinants of low back pain. Curr Pain Headache Rep. 2006;10(5):339-344.

13. Balague F, Dudler J. An overview of conservative treatment for lower back pain. Int J Clin Rheumatol. 2011;6(3):281-290.

14. Jarvik JG. Imaging of adults with low back pain in the primary care setting. Neuroimaging Clin N Amer. 2003:13(2):293-305.

15. Royal College of Radiologists. Making best use of the department of radiology: guidelines for doctors. London: Royal College of Radiologists; 1989

16. Don AS, Carragee E. A brief overview of evidence informed management of chronic low back pain with surgery. Spine J. 2008;8(1):258-265.

17. Bradley WG Jr, Seidenwurm DJ, Brunberg JA, et al. Expert panel on neurological imaging. Low back pain. Reston: American College of Radiology; 2005

18. Jarvik JG, Deyo RA. Diagnostic evaluation of low back pain with emphasis on imaging. Ann Int Med. 2002;137(7):586-597.

19. Gatchel RJ, BoPeng Y, Peters ML, et al. The biopsychosocial approach to chronic pain: scientific advances and future directions. Psychol Bull. 2007;133(4):581-624

20. Lamb SE, Hansen Z, Lall R, et al. Group cognitive behavioral treatment for low back pain in primary care: a randomized controlled trial and cost-effectiveness analysis. Lancet, 2010;375(9718):916-923.

21. Meyer HP. Pain management in primary care: current perspectives. S Afr Fam Pract. 2007;49(7):20-25

22. Burton AK, Waddell G, Tillotson KM, Summerton N. Information and advice to patients with back pain can have a positive effect. Spine (Phila Pa 1976). 1999;24(23):2484-2491.

23. Martell SA, O'Connor PG, Kerns RD, et al. Systematic review: opioid treatment for chronic back pain: prevalence, efficacy, and association with addiction. Ann Intern Med. 2007;146(2):116-127.

24. Dworkin RH, O'Connor AB, Backonja M. Pharmacological management of neuropathic pain: evidence-based recommendations. Pain. 2007:132(3):237-251.

25. Finnerup NB, Otto M, McQuay HJ, et al. Algorithm for neuropatic pain treatment: an evidence based proposal. Pain. 2005;118(3):289-305.

26. Skljarevski V, Desaiah D, Liu-Seifert $H$, et al. Efficacy and safety of duloxetine in patients with chronic low back pain. Spine (Phila Pa 1976). 2010;35(13):E578-E585

27. Hilde G, Hagen KB, Jamtveldt G. Advice to stay active as a single treatment for low back pain and sciatica. [Cochrane review]. In: The Cochrane Library, Issue 2 , 2002. Oxford: Update Software.

28. van Middelkoop M, Rubinstein SM, Verhagen AS, et al. Exercise therapy for chronic nonspecific low back pain. Best Pract Res Clin Rheumatol. 2010;24(2):193-204.

29. Bronfort DC, Haas M, Evans $R$, et al. Evidence-informed management of chronic low back pain with spinal manipulation and mobilization. Spine J. 2008:8(1):213-225.

30. Manheimer E, White A, Berman B, et al. Meta-analysis: acupuncture for low back pain. Ann Intern Med. 2005;142(8):651-663.

31. National Institute for Health and Clinical Excellence. Early management of persistent non-specific low back pain [homepage on the Internet]. 2009 Available from: www.nice.org.uk/CG88

32. Savigny P, Watson P, Underwood M, Guideline Development Group. Management of persistent nonspecific low back pain: summary of the NICE guidance. BMJ. 2009;338:b1805

33. Airaksinen $\mathrm{O}$, Brox $\mathrm{J}$, Cedraschi $\mathrm{C}$, et al. European Guidelines for the management of chronic non-specific low back pain. Eur Spin J. 2006;15 Suppl 2:S192-S300

34. Armon C, Argoff CE, Samuels J, Backonje MM. Assessment: use of epidura steroid injections to treat radicular lumbosacral pain: report of the Therapeutics and Technology Assessment Subcommittee of the American Academy of Neurology. Neurology. 2007:68(10):723-729.

35. Ibrahim T, Tleyjeh M, Gabbar O. Surgical versus nonsurgical treatment of chronic low back pain: a meta-analysis of randomized trials. Int Orth. 2008:32(1):107-113.

36. Evidence-based invasive treatment of chronic musculoskeletal pain. International Association for the Study of Pain; 2009. 